\section{One-Step Multiplex PCR Strategy for Identification of Mutations by SSCP and DNA Sequencing}

\section{BioTechniques 29:234-236 (August 2000)}

One of the most popular PCR-based methods for point mutation detection is single strand conformation polymorphism (SSCP) (5). SSCP is sensitive and relatively simple. It is based on the fact that ssDNA fragments migrate in a non-denaturing polyacrylamide gel according to their primary nucleotide sequence and not their length. DNA fragments with nucleotide sequences that differ by a single nucleotide usually migrate differently. The rate of mutation detection by SSCP varies from gene to gene but is generally in the range of $80 \%-90 \%$. Maximum sensitivity for the detection of sequence variation is usually achieved using PCR fragments of around $300 \mathrm{bp}$ but depends on the optimization of electrophoresis conditions and running temperature (2). For most genes, the small fragment length required for optimal detection of SSCP variants makes it necessary to carry out several individual overlapping reactions to produce the number of fragments necessary to cover the entire sequence to be analyzed. In addition, once a SSCP variation is found and sequenced, it is necessary to confirm the variation in an independent reaction to ensure that the observed mutation is not an error introduced during the PCR.

Here, we describe a simple strategy to produce independent PCR fragments that are ready for direct SSCP analysis and DNA sequencing. The basic strategy is to generate staggered 250-bp PCR fragments from cDNA in independent multiplex reactions that cover the entire target region, which can be subjected to SSCP analysis directly without prior separation of the fragments. Each multiplex reaction will generate non-overlapping 250-bp fragments for SSCP analysis and a larger fragment that contains both of the 250-bp fragments and the intervening sequence between them. Once an SSCP variant is found in a 250-bp multiplex PCR fragment, the larger fragment from the same multi- plex reaction can be isolated from a parallel agarose gel and used for sequence determination to identify the mutation. Although this larger fragment is generated in the same reaction producing the 250-bp fragment with altered SSCP mobility, it can be considered an independent PCR fragment and will confirm the observed SSCP variation as well as identify the mutation.

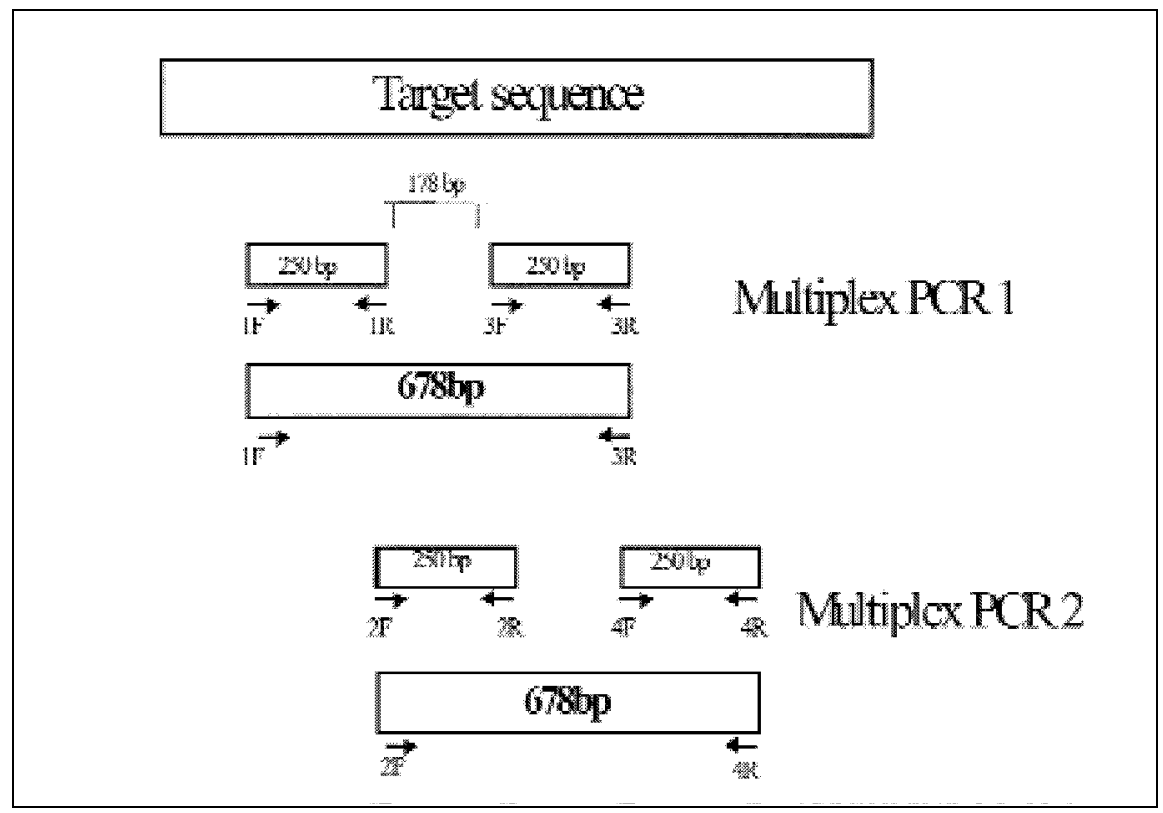

Figure 1. General multiplex PCR strategy.

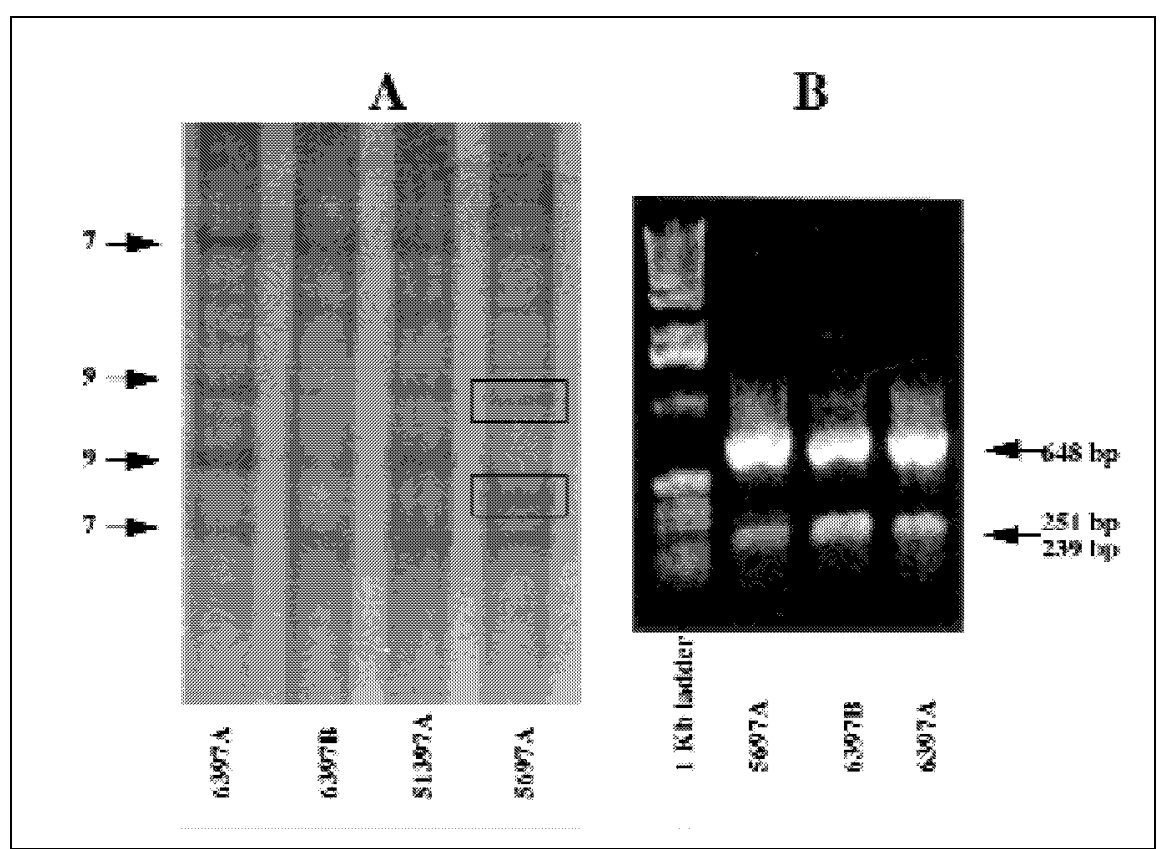

Figure 2. Missense mutation detected in the X-ALD gene. (A) SSCP gel with PCR products from the multiplex reaction with PCR primers 7 and 9 (for primers sequences and positions see Reference 4). SSCP was carried out using $0.75 \times$ MDE (BMA, Rockland, ME, USA) gels prepared with $1 \times$ TBE. Gels were run at $5 \mathrm{~W}$ for $9 \mathrm{~h}$ and silver stained. The gel was initially soaked for $10 \mathrm{~min}$ in a solution containing $10 \%$ methanol and $2.5 \%$ acetic acid, followed by staining with $0.4 \mathrm{~g}$ silver nitrate. Bands with altered mobility in sample 5697A are enclosed in boxes. Arrows indicate the normal bands for PCR products generated with primers 7 and 9. Negative controls were always performed (not shown). (B) 1.2\% agarose gel of multiplex PCR products generated with primers 7 and 9 , showing the larger fragment used for sequence analysis. 
SSCP variants are identified by comparing the migration of corresponding PCR fragments generated from a normal sample and run in the same gel. An ideal scheme for the most efficient mutation analysis of cDNA is diagramed in Figure 1. Only two multiplex reactions are required to cover a target sequence of 892-bp cDNA. In this example, each multiplex reaction uses four PCR primers $(1 \mathrm{~F}, 1 \mathrm{R}$, and $3 \mathrm{~F}, 3 \mathrm{R}$ or $2 \mathrm{~F}, 2 \mathrm{R}$, and $4 \mathrm{~F}, 4 \mathrm{R}$ ) that generate two non-overlapping 250-bp fragments separated by $178 \mathrm{bp}$ and a 678-bp fragment that is the amplification product produced from the proximal forward primer $(1 \mathrm{~F}$ or $2 \mathrm{~F}$ ) and the distal primer (3R or $4 \mathrm{R}$ ). Together, the 250-bp fragments from both multiplex reactions cover the entire 892-bp target sequences with 36-bp overlaps between the PCR fragments in the independent multiplex reactions. An overlap of at least $36 \mathrm{bp}$ is recommended for 18-bp primers to ensure that point mutations in the primer annealing sequence of one fragment will be detected in the fragment from the other reaction. The 678-bp fragment includes the individual $250-\mathrm{bp}$ fragments and the intervening 178-bp sequence. While the PCR primers, fragment sizes and the number of multiplex reactions will vary according to the gene of interest, this strategy is a rapid and efficient method for mutation screens of a large number of samples.

Following the generalized scheme outlined in Figure 1, we have screened for mutations in patients with $\mathrm{X}$-linked Adrenoleukodystrophy (X-ALD) (4). In this instance, four multiplex reactions were carried out to screen for mutations in the X-ALD cDNA (2376 bp). These multiplex reactions generated non-overlapping short PCR fragments ranging from 239-265 bp and longer fragments ranging from $648-882$ bp that contain two of the short fragments and the intervening sequences between them. Figure $2 \mathrm{~A}$ demonstrates a mutation in the $\mathrm{X}$ ALD gene detected by SSCP analysis of a multiplex reaction analyzed without prior separation of fragments (5697A lane, altered bands in boxes). Sequence analyses of the corresponding larger fragment (Figure 2B) isolated from an agarose gel confirmed the SSCP variation and allowed identification of the P543L missense mutation.
A two-step multiplex PCR-SSCP strategy, followed by sequence analysis of a separate PCR fragment generated from the previously identified SSCP variant, has been used to screen for mutations in several different genes $(1,3$, $6)$. These methods require at least two PCR amplifications. The first one is a multiplex reaction that generates multiple fragments of similar sizes for the SSCP analysis. The second PCR generates the fragment for sequencing analysis and confirmation of the mutation detected by SSCP.

The method described here is a onestep strategy that integrates these two reactions into a single step without compromising the confirmation role of the PCR fragment used for sequencing. The co-amplification of the smaller multiplex fragments for PCR analysis and a larger fragment suitable for sequencing in one reaction tube facilitates the analysis of a large number of samples, especially if the source of DNA is limited. Once optimized, this methodology allows efficient and reliable "serial screening" of a large number of samples with no need to subsequently amplify specific PCR fragments for each SSCP variation that was previously found. Using this strategy, we were able to rapidly identify 32 mutations in the X-ALD gene.

\section{REFERENCES}

1.Castresana, J.S., L. Gómez, P. GaruaMiguel, A. Queizan and A. Pestana. 1997. Mutational analysis of the p16 gene in human neuroblastoma. Mol. Carcinog. 18:129-133.

2.Fan, E., D.B. Levin, B.M. Glickman and D.M. Logan. 1993. Limitations in the use of SSCP analysis. Mutat. Res. 288:85-92.

3.Kneppers, A.L.J., P.P. Deutz-Terlouw, J.T. Dunnen, G.J.B. van Ommen and E. Bakker. 1995. Point mutations screening for 16 exons of the dystrophin gene by multiplex single-strand conformation polymorphism analysis. Hum. Mutat. 5:235-242.

4.Lachtermacher, M.B.R., H.N. Seuanez, A.B. Moser, H.W. Moser and K.D. Smith. 2000. Determination of 30 X-linked Adrenoleukodystrophy mutations, including 15 not previously described. Hum. Mutat. 15:348-353.

5.Orita, M., H. Iwahana, H. Kanazava, K. Hayashi and T. Sekyia. 1989. Detection of polymorphism of human DNA by gel electrophoresis as single strand conformation polymorphisms. Proc. Natl. Acad. Sci. USA 86:2766-2770.

6.Philips, K-A., K. Nichol, H. Ozcelik, J.
Knight, S.J. Done, P.J. Goodwin and I.L. Andrulis. 1999. Frequency of p53 mutations in breast carcinomas from Ashkenazi Jewish carriers of BRCA1 mutations. J. Natl. Cancer Inst. 91:469-473.

Address correspondence to Dr. Marcia Lachtermacher B. Rocha, NCI/FCRDC, Laboratory of Genomic Diversity, Frederick, MD 21702-1201, USA. e-mail: rocham @ncifcrf.gov

Received 15 December 1998; accepted 27 March 2000.

Marcía B.R.

Lachtermacher ${ }^{1,2}$, Héctor $\mathbf{N}$. Seuanez ${ }^{3,4}$, Hugo W. Moser ${ }^{1,5}$ and Kirby D. Smith ${ }^{\mathbf{1 , 5}}$

${ }^{1}$ Kennedy Krieger Institute

Baltimore, MD, USA

${ }^{2}$ Universidade Federal

Fluminense

Niterói, Brazil

${ }^{3}$ Instituto Nacional de Câncer

Rio de Janeiro, Brazil

${ }^{4}$ Universidade Federal do Rio de Janeiro

Rio de Janeiro, Brazil

${ }^{5}$ Johns Hopkins School of

Medicine

Baltimore, MD, USA
RNA Amplification Technique, NASBA, Also Amplifies Homologous Plasmid DNA in NonDenaturing Conditions

BioTechniques 29:236-240 (August 2000)

NASBA is described as a highly sensitive amplification method specific for RNA. It permits the detection of eukaryotic gene transcription even if the RNA samples contain traces of genomic DNA (2). Recently, NASBA was de- 